\title{
XLVI. The viscosity of water
}

\section{Richard Hosking B.A.}

To cite this article: Richard Hosking B.A. (1909) XLVI. The viscosity of water, Philosophical Magazine Series 6, 17:100, 502-520, DOI: 10.1080/14786440408636626

To link to this article: http://dx.doi.org/10.1080/14786440408636626

$$
\text { 册 Published online: } 21 \text { Apr } 2009 .
$$

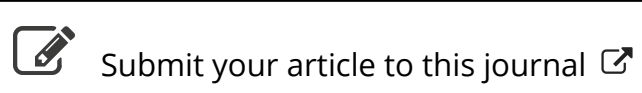

\footnotetext{
Џlll Article views: 3
}

Q View related articles ¿

Citing articles: 2 View citing articles $\square$ 
multiplied by $r_{1} / r_{2}$. In this instance $r_{1} / r_{2}$ was $1 \cdot 61$ and $\mathrm{E} / \mathrm{C}=r_{2} / r_{1} \cdot \theta_{1} / \theta_{2}=\mathbf{2} \cdot \mathbf{4 8 5}$ or $\mathrm{E}=30,800,000$ and Poisson's ratio $=\cdot 243$.

A tension-test on a similar piece of tube gave $\mathrm{E}=30,700,000$.

In addition to its applications for bending and twisting the apparatus may be used for testing a variety of cases of combined stress if a pump is added to give a fluid pressure in the interior of tubes.

The accompanying diagram (Pl. XI. fig. 10) shows the results of tests to failure of bicycle-tubing when subjected to (I.) bending, (II.) twisting, and (III.) twisting combined with a uniform bending moment.

XLVI. The Viscosity of Water.

By Richard Hosking, B.A. (Camb.).*

[Plate XIII.]

TN my previous experiments on the determination of viscosity by the efflux method $\dagger, I$ have always arranged to have the rate of flow of liquid in the capillary tube very small. The kinetic energy correction in the well-known reduction formula was thus always small in comparison with the first term. In the present experiments, however, I have purposely increased this rate of flow in order to test the formula in cases where the kinetic energy correction is much greater. The glischrometer used in these experiments was of the same form as those previously used by me, but the bulbs were larger. It is shown in fig. 1. At $a, b, c$, and $d$, platinum wires are inserted, which are almost touching inside the tubes. The capillary $\mathrm{C}$ is fitted to the limbs by rubber bands. The volume of the bulb $\mathrm{R}$ at $0^{\circ} \mathrm{C}$. is 10.2801 ces., and that of $\mathrm{L}$ at the same temperature 10.3201 ccs. Four capillaries were carefully selected for separate use in the glischrometer, and their ends were ground with fine emery, in a lathe. Their lengths, measured directly with callipers, were $5 \cdot 570,6 \cdot 494,5 \cdot 408$, and $6 \cdot 456$ cms. respectively at $0^{\circ}$ C., and their radii approximately were $\cdot 019, \cdot 019, \cdot 020, \cdot 020 \mathrm{~cm}$. respectively, at $0^{\circ} \mathrm{C}$. (The exact determination of the equivalent radius of each capillary was made at the end of all the experiments. It involved the cutting up of the tube and the careful measurement of the sections.) Sufficient freshly distilled water was put in to fill up the glischrometer from $b$ to $c$.

* Communicated by the Author. Read before the Royal Society of N. S. Wales, June 3, 1908.

+ Phil. Mag. March 1900; May 1902; May 1904. 
The ends A and B were connected to the reservoir of compressed air or the outside air by means of three-way taps. Measurements of viscosity were taken first with the water flowing out of $R$ into $L$, and secondly with the water

Fig. 1.

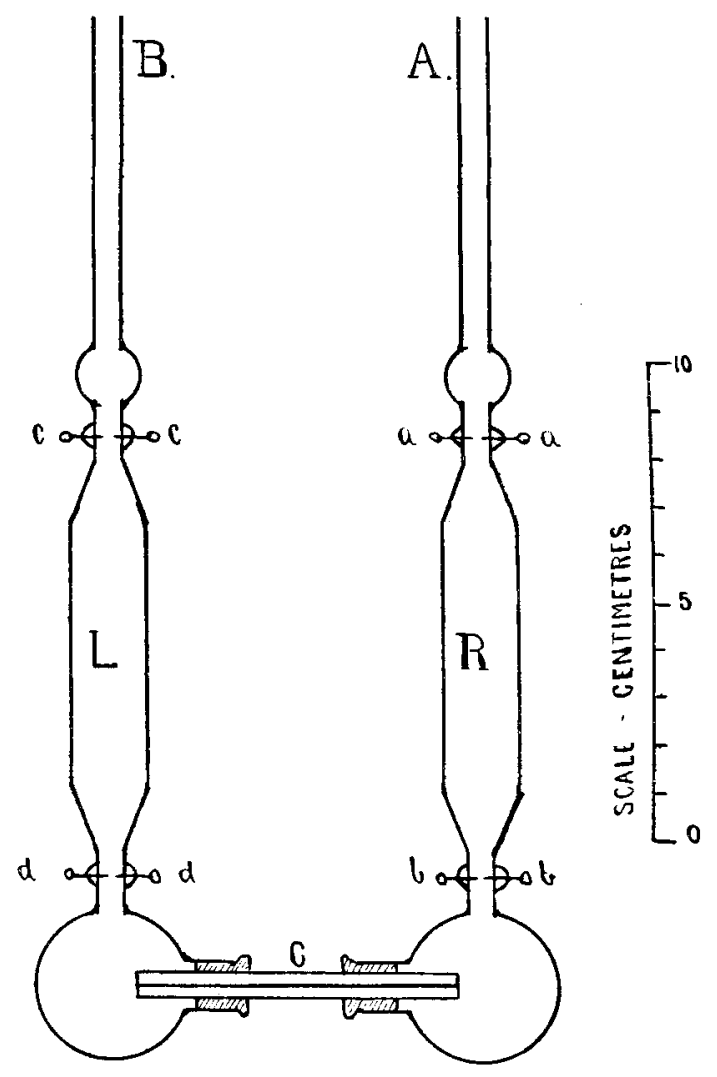

flowing in the opposite way, under the pressure of air in the reservoir. The average of the two determinations was taken as an absolute value of the viscosity. The pressure was measured by means of (1) a water manometer $200 \mathrm{cms}$. long, (2) a mercury manometer in cases where the pressure was greater than $200 \mathrm{cms}$. of water.

To facilitate the reading of the water manometer, a pair of small parallel mirrors was attached to each of the arms. These were inclined so as to make an angle of $45^{\circ}$ with the vertical manometer scale. One was fixed near the centre of 
the arm, and opposite a telescope; the other could be moved along the arm and clamped in front of the water surface. When the mirrors had been set, it was thus possible to read off the positions of the two water surfaces by means of the telescope, at the same instant; for both images were arranged to be side by side in the field. The pressures employed varied between the limits $100 \mathrm{cms}$. (water) and $42 \mathrm{cms}$. (mercury).

The time of flow was in most cases very short, the average being about one minute, but in extreme cases it was as low as 22 seconds. Special means had to be employed to register the time intervals correctly. The chronograph used was kindly supplied by the Sydney Observatory. It consisted of two electromagnets side by side. The armatures were provided with needles. Paper tape was fed through rollers immediately over the needle points at the rate of about $5 \mathrm{cms}$. per second. A special spring enabled the needles to travel forward a little on piercing the tape, and prevented the tearing of the tape. One needle was used for recording seconds by direct reference, through electrical contacts, to a standard clock. The other needle punctured the tape when a key was pressed at the transits of the meniscus in the glischrometer at the points $a$ and $b$ in the one case; or at $c$ and $d$ in the other. These transits were observed always through telescopes.

The procedure in determining the viscosity was as follows. The bath temperature was arranged to be as close to the desired temperature as possible, and the beating flame was adjusted. The pressure of air in the reservoir was raised or lowered to the proper level. Double readings of pressure, time of flow, and temperature were taken. The pressure was next altered, and more readings were taken. In most cases, the determinations were repeated. Another capillary was then placed in position in the glischrometer and the series was repeated.

The Reduction Formula.-In the Journal and Proceedings of the Royal Society of New South Wales are published three most important papers by G. H. Knibbs*, dealing with the history, theory, and determination of the viscosity of water by the efflux method. Knibbs has shown that the reduction formula is

$$
\eta_{t}=\frac{\pi \mathrm{R}^{1}}{8 \mathrm{VI}\left(1+n_{\mathrm{L}}^{\mathrm{R}}\right)} g \cdot \rho h \cdot \mathrm{T}-\frac{m \cdot \delta \cdot \mathrm{V}(1+2 k t)}{8 \pi \mathrm{L}\left(1+n^{\mathrm{L}}\right) \mathrm{T}}
$$

* Vols. xxix., xxx., and $x x x i$. 
In this formula $L$ is the length and $R$ the radius of the capillary, and $\mathrm{T}$ the time taken for volume $\mathrm{V}$ of the liquid of density $\delta$ to flow through the capillary under a pressure $g \rho / ; n \mathrm{R}$ is a small length of tube producing a loss of pressure equivalent to that arising from the friction at the ends, its value must be calculated for each series of experiments; $m$ is the numerical factor in the kinetic energy correction, which has a theoretical value of $1 \cdot 12$, but which has a practical value which must be determined. The factor $m$ has been neglected in so many recent determinations of viscosity, that it is worth the while to repeat the information given by Knibbs respecting it. In 1860 Neumann deduced the value $m=1$, and Jacobsen used it in his 'Introduction to Hremodynamics' (1860). Hagenbach deduced the value $m=1$ in the same year. Reynolds (following Bernoulli) in 1883 used the value $m={ }_{2}^{1}$. Conette in 1890 independently obtained the value $m=1$. Boussinesq (1891) obtained a more accurate value $m=1 \cdot 12$. Gartenmeister stated (1890) that Finkener had in an unpublished treatise shown that Conette's value was the correct (?) one. Wilberforce (1891) pointed out the defect in Hagenbach's reasoning, and he used the value $m=1$. Knibbs has shown that theoretically Neumann's correction as deduced by Boussinesq is correct, and that experimentally its value varies considerably. Knibbs has deduced values of $m$ from Jacobsen's results, and stated that individual results show how, even under circumstances in which uniformity might be expected, it is not realized; and that if the correction be of sensible magnitude, the deduced viscosity is, to the extent of this uncertainty, unreliable.

Determination of $\mathrm{m}$ and $\mathrm{n}$.- Preliminary experiments were made in order to obtain correct (experimental) values for the constants $m$ and $n$. The temperatures were kept as close to $50^{\circ} \mathrm{C}$. as possible, and, under different pressures, the times of flow were recorded. The pressures were reduced to equivalent pressures at $50^{\circ} \mathrm{C}$. Knibbs has shown that the reduction formula, for experiments carried out at a certain temperature, may be expressed in the form

$$
\begin{aligned}
& \mathrm{C}+c / \mathrm{T}=\rho / \mathrm{T}, . . \quad . \quad . \quad . \\
& \text { where } \quad \mathrm{C}=\eta_{t}(1+n \mathrm{R} / \mathrm{L}) \frac{8 \mathrm{~V} \mathrm{~L}}{\pi g \mathrm{R}^{4}}, . . . \\
& \text { and } \quad c=\frac{m \delta}{g \pi^{2}} \mathrm{~V}^{2}(1+2 k t) . \quad . \quad . \quad .
\end{aligned}
$$

Equation (2) is that of a straight line such that if $1 / \mathrm{T}$ be taken as abscissa, and corresponding values of $\rho h \mathrm{~T}$ as ordinates, the line passing through the points so determined 
506 Mr. R. Hosking on the Viscosity of Water.

will intersect the axis of ordinates at a distance $\mathrm{C}$ from the origin, and make with the axis of abscissæ an angle whose tangent is $c$. When $c$ has been obtained $m$ is deduced by means of equation (4).

Equation (3) may be written in the form

$$
\frac{\mathrm{C} \pi g \mathrm{R}^{4}}{8 \mathrm{VL}}=\eta_{t}\left(1+n \frac{\mathrm{R}}{\mathrm{L}}\right) \text {. }
$$

The left-hand side of the equation will have different values for different capillaries in the glischrometer.

Calling the left side $\mathrm{K}$ we have

where $l=n \eta_{l-}$

$$
\mathrm{K}=\eta_{t}+n \eta_{t} \mathrm{R} / \mathrm{L} \text { or } \mathrm{K}=\eta_{t}+l \mathrm{R} / \mathrm{L}, \quad \text {. }
$$

This is the equation of a straight line such that if values of $R / L$ be taken as abscissæ, and corresponding values of $\mathrm{K}$ as ordinates, the line passing through such points will intersect the axis of ordinates at a distance $\eta_{t}$ from the origin, and make an angle with the axis of abscissæ whose tangent is $k$. From the value of $k$ obtained in this way, $n$ is at once deduced.

\begin{tabular}{|c|c|c|c|c|c|c|}
\hline $\begin{array}{l}\text { Temp. } \\
\left(t^{\circ} \mathrm{C} .\right)\end{array}$ & $\begin{array}{c}\text { Manometer } \\
\text { Reading }(h) \\
\text { chis. }\end{array}$ & $\begin{array}{c}\text { Temp. of } \\
\text { Manometer } \\
{ }^{\circ} \mathrm{C} .\end{array}$ & $\begin{array}{c}\text { Time of } \\
\text { Flow }(\mathbf{T}) \\
\text { secs. }\end{array}$ & $\begin{array}{c}\text { Bulb } \\
\text { being } \\
\text { emptied. }\end{array}$ & $\begin{array}{c}\rho h \mathrm{~T} \\
\text { (reduced to } \\
\left.50^{\circ}\right) \times 10^{-3}\end{array}$ & $\frac{1}{T}$ \\
\hline 5005 & * $108 \cdot 29$ & $22 \cdot 9$ & $71 \cdot 38$ & $\mathbf{R}$ & $7 \cdot \div 13$ & .01401 \\
\hline $50 \cdot 03$ & $108 \cdot 27$ & 0 & $71 \cdot 46$ & L & 7.718 & .01400 \\
\hline $50 \cdot 00$ & $108 \cdot 31$ & 2 & $71 \cdot 45$ & $\mathbf{R}$ & $7 \cdot 707$ & .01400 \\
\hline 5000 & $108 \cdot 31$ & & $71 \cdot 33$ & $\mathrm{~L}$ & $7 \cdot 697$ & .01402 \\
\hline $50 \cdot 10$ & $150 \cdot 28$ & $26 \% 0$ & $54 \cdot 34$ & $\vec{R}$ & $8 \cdot 145$ & .01840 \\
\hline $50 \cdot 10$ & $150 \cdot 30$ & & $54 \cdot 37$ & $\mathbf{L}$ & $8 \cdot 150$ & .01839 \\
\hline $50 \cdot 00$ & 196.20 & $22 \cdot 9$ & 43.98 & $\overrightarrow{\mathrm{R}}$ & 8.598 & .02274 \\
\hline $50 \cdot 00$ & $196 \cdot 14$ & & $43 \cdot 74$ & $\mathbf{L}$ & $8 \cdot 546$ & .02286 \\
\hline $50 \cdot 00$ & $196 \cdot 10$ & $24 \cdot 2$ & $44 \cdot 03$ & $\mathbf{R}$ & $8 \cdot 600$ & .02272 \\
\hline $50 \cdot 00$ & $196 \cdot 17$ & & $43 \cdot 70$ & $\mathbf{L}$ & $8 \cdot 540$ & .02288 \\
\hline $50 \cdot 04$ & $+20 \cdot 30$ & $23 \cdot 9$ & $33 \cdot 58$ & $\vec{R}$ & $9 \cdot 239$ & .02978 \\
\hline 50.03 & $20 \cdot 30$ & ," & $33+52$ & $\mathbf{I}$ & $9 \cdot 220$ & .02984 \\
\hline $50 \cdot 02$ & $20 \cdot 30$ & 13 & $33 \cdot 58$ & $\mathbf{R}$ & $9 \cdot 236$ & .02978 \\
\hline $50 \cdot 01$ & $20 \cdot 30$ & & $33 \div 53$ & $\mathbf{L}$ & $9 \cdot 232$ & .02985 \\
\hline $50 \cdot 10$ & $25 \cdot 40$ & $26 " 0$ & $28 \cdot 35$ & $\mathbf{R}$ & $9 \cdot 764$ & .03527 \\
\hline $50 \cdot 10$ & $24 \cdot 40$ & & $29 \cdot 24$ & $\mathbf{L}$ & $9 \cdot 674$ & $\cdot 03420$ \\
\hline $50 \cdot 05$ & $30 \cdot 38$ & 2399 & 24.78 & $\mathbf{R}$ & $10 \cdot 204$ & .04035 \\
\hline $50 \cdot 06$ & $30 \cdot 38$ & ", & 24.93 & $\mathrm{~L}$ & 10.267 & $\cdot 04011$ \\
\hline $50 \cdot 07$ & $30 \cdot 38$ & , & $2+72$ & $\bar{R}$ & $10 \cdot 184$ & .04045 \\
\hline $50 \div 08$ & $30 \cdot 38$ & $n$ & $24 \cdot 95$ & $\mathbf{L}$ & $10 \cdot 280$ & .04008 \\
\hline $50 \cdot 00$ & $35 \cdot 40$ & $22 \cdot 9$ & $22 \cdot 32$ & $\mathrm{R}$ & 10.701 & .04480 \\
\hline $50 \cdot 00$ & $35 \cdot 40$ & ,* & $22 \cdot 38$ & $\mathrm{I}$ & 10726 & $\cdot 04468$ \\
\hline $50 \cdot 00$ & $35 \cdot 40$ & $2 t$ & $22 \cdot 28$ & $\mathrm{R}$ & $10 \cdot 683$ & .04488 \\
\hline $50 \cdot 00$ & $35 \cdot 40$ & & $22 \cdot 38$ & $\mathrm{~L}$ & $10 \cdot 726$ & .04468 \\
\hline $50 \cdot 10$ & $40 \cdot 40$ & $22 \cdot 9$ & $20 \cdot 36$ & $\vec{R}$ & $11 \cdot 171$ & .04912 \\
\hline $50 \cdot 10$ & $40 \cdot 40$ & ," & $21 \cdot 40$ & $\mathbf{L}$ & 11.737 & .04673 \\
\hline $50 \cdot 10$ & $40 \cdot 40$ & ", & 20.39 & $\mathbf{R}$ & $11 \cdot 188$ & .04904 \\
\hline $50 \cdot 10$ & $40 \cdot 40$ & , & $21 \cdot 40$ & $\mathrm{~L}$ & 11.737 & $\cdot 04673$ \\
\hline
\end{tabular}

Typical Observations with Tube I. in Glischrometer.

* Water Manometer.

+ Mercury Manometer. 
Typical Observations with Tube II. in Glischrometer.

\begin{tabular}{|c|c|c|c|c|c|c|}
\hline $\begin{array}{l}\text { Temp. } \\
\left(t^{\circ} \mathrm{C} .\right) \text {. }\end{array}$ & $\begin{array}{c}\text { Manometer } \\
\text { Reading }(h) \\
\text { ems. }\end{array}$ & $\begin{array}{l}\text { Temp. of } \\
\text { Manometer } \\
\text { o. }\end{array}$ & $\begin{array}{c}\text { Time of } \\
\text { Flow (T) } \\
\text { sees. }\end{array}$ & $\begin{array}{c}\text { Bulb } \\
\text { being } \\
\text { emptied. }\end{array}$ & $\begin{array}{c}\rho h \mathrm{~T} \\
(\text { reduced to } \\
\left.50^{\circ}\right) \times 10^{-3}\end{array}$ & $\frac{1}{T}$ \\
\hline 50.08 & ${ }^{*} 108.41$ & $26 \cdot 7$ & $80 \cdot 41$ & $\mathbf{R}$ & $8 \cdot 716$ & .01244 \\
\hline $50 \cdot 08$ & $108 \cdot 25$ & 80 & $80 \cdot 27$ & $\mathrm{~L}$ & $8 \cdot 676$ & .01246 \\
\hline 50.08 & $108 \cdot 13$ & ", & $80 \cdot 81$ & $\mathrm{R}$ & $8 \cdot 729$ & .01237 \\
\hline 50.08 & $108 \cdot 15$ & & $80 \cdot 48$ & $\mathrm{~L}$ & $8 \cdot 690$ & .01242 \\
\hline $50 \cdot 05$ & $151 \cdot 12$ & $26 \cdot 7$ & $60 \cdot 40$ & $\mathbf{R}$ & $9 \cdot 113$ & 01656 \\
\hline $50 \cdot 06$ & $151 \cdot 02$ & ", & $60 \cdot 30$ & $\mathbf{L}$ & $9 \cdot 084$ & 01658 \\
\hline $50 \cdot 07$ & $150 \cdot 96$ & ," & $60 \cdot 40$ & $\bar{R}$ & $9 \cdot 118$ & .01656 \\
\hline $50 \cdot 07$ & 150.96 & & $60 \cdot 18$ & $\mathrm{I}_{s}$ & $9 \cdot 065$ & .01661 \\
\hline 50.02 & $197 \cdot 10$ & $26 \cdot 7$ & 48.58 & $\mathbf{R}$ & $9 \cdot 549$ & 02058 \\
\hline $50 \cdot 04$ & $197 \cdot 20$ & $"$ & $48 \cdot 15$ & L & $9 \cdot 470$ & 02077 \\
\hline $50 \cdot 05$ & $197 \cdot 04$ & $n$ & $48 \cdot 45$ & $\mathrm{R}$ & $9 \cdot 531$ & 02064 \\
\hline $50 \cdot 05$ & 197.08 & & $48 \cdot 15$ & $\mathrm{~L}$ & $9 \cdot 468$ & .02077 \\
\hline 5000 & $120 \cdot 40$ & 267 & $36 \cdot 79$ & $\mathrm{R}$ & $10 \cdot 153$ & .02718 \\
\hline 5000 & 2038 & " & $36 \cdot 68$ & $\mathrm{~L}$ & $10 \cdot 113$ & 02726 \\
\hline $50 \cdot 00$ & $20 \cdot 38$ & ", & 36.93 & $\mathrm{R}$ & $10 \cdot 180$ & $\cdot 02708$ \\
\hline $50 \cdot 00$ & $20 \cdot 38$ & & 36.85 & $\mathbf{I}$ & $10 \cdot 157$ & .02714 \\
\hline $50 \cdot 10$ & $30 \cdot 42$ & $26 \cdot 7$ & 27.00 & $\mathrm{R}$ & $11 \cdot 145$ & 03703 \\
\hline $50 \cdot 10$ & $30 \cdot 42$ & , & $27 \cdot 16$ & $\overline{\mathrm{L}}$ & $11 \cdot 200$ & .03682 \\
\hline $50 \cdot 02$ & $30 \cdot 42$ & 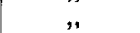 & $27 \cdot 29$ & $\boldsymbol{R}$ & 11.237 & .03664 \\
\hline $50 \cdot 02$ & 30.42 & & $26 \cdot 97$ & $\mathrm{I}$ & $11 \cdot 116$ & .03707 \\
\hline$(50 \cdot 10$ & $40 \cdot 39$ & $21 \cdot 9$ & 22.07 & $\bar{R}$ & $12 \cdot 107$ & .04552 \\
\hline $50 \cdot 10$ & $40 \cdot 39$ & $"$ & 21.97 & L & $12 \cdot 053$ & .04511 \\
\hline $50 \cdot 10$ & $40 \cdot 39$ & ", & $22 \cdot 17$ & $\mathrm{R}$ & $12: 152$ & 04564 \\
\hline$\left(50^{\circ}\right] 10$ & $40 \cdot 39$ & " & 21.91 & $\mathrm{~L}$ & $12 \cdot 020$ & .04585 \\
\hline
\end{tabular}

* Water Manometer.

+ Mercury Manometer.

Typical Observations with Tube III. in Glischrometer.

\begin{tabular}{|c|c|c|c|c|c|c|}
\hline $\begin{array}{l}\text { Temp. } \\
\left(t^{\circ} \mathrm{C} .\right) .\end{array}$ & $\begin{array}{c}\text { Mancmeter } \\
\text { Reading }(h) \\
\text { cms. }\end{array}$ & $\begin{array}{c}\text { Temp. of } \\
\text { Manometer } \\
{ }^{\circ} \mathrm{C} .\end{array}$ & $\begin{array}{c}\text { Time of } \\
\text { Flow (T) } \\
\text { secs. }\end{array}$ & $\begin{array}{c}\text { Bulb } \\
\text { being } \\
\text { emptied. }\end{array}$ & $\begin{array}{c}\rho h \mathrm{~T} \\
(\text { reduced to } \\
\left.50^{\circ}\right) \times 10^{-3}\end{array}$ & $\frac{1}{\mathrm{~T}}$ \\
\hline 5007 & $* 108.28$ & 235 & $54 \cdot 56$ & $\mathbf{R}$ & 5.897 & 01833 \\
\hline 5007 & $108 \cdot 18$ & 00 & $54 \cdot 60$ & $\mathrm{~L}$ & 5.897 & .01831 \\
\hline $50 \cdot 07$ & $108 \cdot 15$ & $"$ & 54.57 & $\mathbf{R}$ & 5.891 & 018.33 \\
\hline $150 \cdot 07$ & $108 \cdot 17$ & & $54 \cdot 56$ & $\mathbf{L}$ & $5 \cdot 893$ & $.0183 ?$ \\
\hline$(50 \cdot 20$ & $151 \cdot 14$ & $23 \cdot 4$ & $41 \cdot 69$ & $\mathrm{R}$ & $6 \cdot 316$ & $\cdot 02399$ \\
\hline $50 \cdot 24$ & $151 \cdot 14$ & $"$ & $41 \cdot 64$ & $\mathrm{~L}_{i}$ & $6: 325$ & .02401 \\
\hline $50 \cdot 22$ & $151 \cdot 08$ & ", & $41 \cdot 72$ & $\mathbf{R}$ & $6 \cdot 321$ & $\cdot 02397$ \\
\hline$(50.22$ & $151 \cdot 08$ & & 41.58 & L & $6 \cdot 300$ & -02404 \\
\hline 50.20 & $197 \cdot 40$ & 2355 & 34.00 & $\mathbf{R}$ & 6705 & 02941 \\
\hline $50 \cdot 10$ & $197 \cdot 30$ & & 33.93 & L & $6 \cdot 690$ & 02948 \\
\hline $50 \cdot 10$ & $197 \cdot 14$ & & 33.94 & $\mathrm{R}$ & $6 \cdot 684$ & .02947 \\
\hline$(50 \cdot 10$ & $197 \cdot 26$ & & 33.95 & L & $6 \cdot 691$ & .02946 \\
\hline 5007 & +2030 & $23 \cdot 2$ & 26.55 & $\mathrm{R}$ & $7 \cdot 311$ & 03767 \\
\hline $50 \cdot 07$ & $20 \cdot 30$ & $"$ & $26 \cdot 47$ & $\mathrm{~L}$ & $7 \cdot 290$ & .03779 \\
\hline $50 \cdot 07$ & $20 \cdot 30$ & 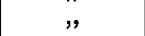 & 26.52 & $\mathbf{R}$ & $7 \cdot 303$ & .03772 \\
\hline $50 \cdot 07$ & $20 \cdot 30$ & & $26 \cdot 44$ & L & $7 \cdot 282$ & 03783 \\
\hline 50.08 & $30 \cdot 39$ & $23 \cdot 9$ & $19 \cdot 98$ & $\mathrm{R}$ & $8 \cdot 237$ & 05006 \\
\hline 50.08 & $30 \cdot 39$ & $"$ & $19 \cdot 86$ & I & $8 \cdot 187$ & .05036 \\
\hline $50 \cdot 08$ & $30 \cdot 39$ & 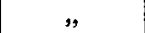 & $19 \cdot 98$ & $\mathbf{R}$ & $8 \cdot 237$ & 05006 \\
\hline 50.08 & $30 \cdot 39$ & & $19 \cdot 82$ & $\mathbf{L}$ & $8 \cdot 17 \mathrm{I}$ & .05046 \\
\hline$[50 \cdot 10$ & $40 \cdot 42$ & 242 & $17: 51$ & $\mathrm{R}$ & $9 \cdot 606$ & 05711 \\
\hline $50 \cdot 10$ & $40 \cdot 42$ & $\because$ & 16.43 & L & $9 \cdot 016$ & .06088 \\
\hline $50 \cdot 10$ & $40 \cdot 42$ & $m$ & $J 7 \cdot 54$ & $\mathrm{R}$ & $9 \cdot 624$ & .05701 \\
\hline$(50 \cdot 10$ & $40 \cdot 42$ & & $16 \cdot 22$ & $L$ & 9.900 & .06165 \\
\hline
\end{tabular}

* Water Manometer. † Mereury Manometer. 
Typical Observations with Tube IV. in Glischrometer.

\begin{tabular}{|c|c|c|c|c|c|c|}
\hline $\begin{array}{l}\text { Temp. } \\
\left(t^{\circ} \mathrm{C} .\right)\end{array}$ & $\begin{array}{l}\text { Manómeter } \\
\text { Reading }(h) \\
\text { cms. }\end{array}$ & $\begin{array}{l}\text { Temp. of } \\
\text { Manometer } \\
{ }^{\circ} \mathrm{C} .\end{array}$ & $\begin{array}{l}\text { Time of } \\
\text { Flow (T) } \\
\text { secs. }\end{array}$ & $\begin{array}{c}\text { Bulb } \\
\text { being } \\
\text { emptied. }\end{array}$ & $\begin{array}{c}\rho h \mathrm{~T} \\
\text { (reduced to } \\
\left.50^{\circ}\right) \times 10-3 \text {. }\end{array}$ & $\frac{1}{T}$. \\
\hline $\int 50 \cdot 10$ & $* 108.08$ & 267 & $61 \cdot 26$ & $\mathbf{R}$ & $6 \cdot 614$ & .01632 \\
\hline $50 \cdot 10$ & $108^{\circ}$ & & $60^{\circ}$ & $\mathrm{L}$ & 6.561 & .01645 \\
\hline $50 \cdot 10$ & $108 \cdot 09$ & , & $61 \cdot 83$ & $\mathrm{R}$ & $6 \cdot 675$ & .01617 \\
\hline $50 \cdot 10$ & $108 \cdot 10$ & & $61 \cdot 21$ & $\mathrm{~L}$ & 6607 & .01634 \\
\hline 50.04 & $150 \cdot 97$ & $26 \cdot 7$ & $46 \cdot 49$ & $\mathrm{R}$ & 6.995 & .02151 \\
\hline $50 \cdot 01$ & 151.07 & $"$ & $46 \cdot 30$ & L & 6.967 & .02160 \\
\hline $50 \cdot 00$ & $150 \cdot 96$ & , & 46.50 & $\mathrm{R}$ & $6 \cdot 990$ & .02150 \\
\hline 5000 & $150 \cdot 96$ & & $46 \cdot 30$ & $\mathrm{~L}$ & $7 \cdot 955$ & .02160 \\
\hline $50 \cdot 00$ & $197 \cdot 17$ & 26.0 & $37 \cdot 44$ & $\vec{R}$ & $7 \cdot 348$ & 02671 \\
\hline $50-00$ & 197.07 & 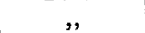 & $37 \cdot 35$ & $\mathrm{I}$ & $\tau \cdot 326$ & .02671 \\
\hline $50 \cdot 00$ & 19704 & , & $37 \cdot 54$ & $\mathbf{R}$ & $7 \cdot 359$ & 02664 \\
\hline $50 \cdot 00$ & 196.98 & & $37 \cdot 37$ & $\overrightarrow{\mathbf{L}}$ & $7 \cdot 326$ & .02677 \\
\hline $50 \cdot 10$ & $+20 \cdot 92$ & 257 & $28 \cdot 25$ & $\mathbf{R}$ & 8.023 & $\cdot 03539$ \\
\hline $50 \cdot 10$ & $20 \cdot 96$ & ", & $28 \cdot 00$ & $\mathrm{I}$ & 7.998 & 03571 \\
\hline $50 \cdot 10$ & $20 \cdot 92$ & 70 & 2832 & $\mathrm{R}$ & $8 \cdot 042$ & 03531 \\
\hline $50 \cdot 10$ & 20.92 & & $28 \cdot 10$ & $\mathrm{~L}$ & $7 \cdot 980$ & 03559 \\
\hline $50 \cdot 10$ & 30.98 & $25 \cdot 7$ & $21 \cdot 30$ & $\mathbf{R}$ & 8.954 & .04698 \\
\hline $50 \cdot 10$ & $30 \cdot 99$ & ," & 22.80 & $\mathrm{I}$ & 9.594 & .04386 \\
\hline $50 \cdot 10$ & $31 \cdot 00$ & $"$ & $21 \cdot 40$ & $\mathrm{R}$ & $9 \cdot 010$ & $.0467 \mathrm{I}$ \\
\hline$(50 \cdot 10$ & $31 \cdot 00$ & & $22 \cdot 58$ & L & $9 \cdot 498$ & .04429 \\
\hline 50.00 & $40 \cdot 47$ & $25 \cdot 7$ & 2078 & $\bar{R}$ & $11 \cdot 378$ & .04812 \\
\hline $50 \cdot 00$ & $40 \cdot 47$ & ", & $21 \cdot 33$ & $\mathrm{~L}$ & $11 \cdot 682$ & 04688 \\
\hline 5000 & $40 \cdot 47$ & ", & $20 \cdot 43$ & $\mathrm{R}$ & $11 \cdot 185$ & 04895 \\
\hline 150.00 & $40 \cdot 47$ & 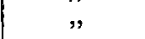 & 21.05 & $\mathrm{I}$ & 11.530 & .04750 \\
\hline
\end{tabular}

* Water Manometer.

+ Mereury Manometer.

The numbers in columns 6 and 7 were used to obtain the curves given in figs. $2,3,4, \& 5$ (Pl. XIII.). It will be noticed that for a considerable distance the lines are straight, indicating constant values for $c$ in equation (2), and therefore for $m$ for the particular capillary. This constancy is remarkable when we consider the enormous speed with which the water is forced through the tubes in many cases. The individual observations show also that there is no variation in any particular case, and that the value of $m$ in the general formula can be relied upon, when determined in this way. There is, therefore, no necessity to keep the kinetic energy correction small in comparison with the first term in determining viscosities by the efflux method, provided, of course, that the time of flow and pressure can be measured with sufficient accuracy. This will be shown later, where values have been worked out. In certain curves it will also be noticed that at a particular point, there is an abrupt change in the direction of the line, indicating either a largely increased value for $m$, or a change in the nature of the flow. "This is most marked in both the curves for Tube IV. and in one of the curves for Tube III.

The following values for $\mathrm{C}$ and $c$ in equation (2) were 
obtained from the curves, and the corresponding values of $m$ were deduced by equation (4).

\begin{tabular}{|c|c|c|c|c|c|c|c|c|}
\hline & \multicolumn{2}{|c|}{ Tube I. } & \multicolumn{2}{|c|}{ Tube II. } & \multicolumn{2}{|c|}{ Tube III. } & \multicolumn{2}{|c|}{ Tube IV. } \\
\hline & R to $\mathrm{L}$. & L to $R$. & $R$ to $\mathrm{L}$. & L to $R$ & 'R to $\mathrm{L}$ & L to $R$. & $\mathbf{R}$ to $\mathbf{L}$ & L to $\mathbf{R}$. \\
\hline $\mathrm{C}$ & 6350 & 6330 & 7400 & 7440 & 4570 & 4570 & 5400 & 5390 \\
\hline $10^{-4} \times c \ldots$ & $9 \cdot 60$ & $9 \cdot 81$ & $10 \cdot 00$ & 990 & $7 \cdot 78$ & 7.78 & $7 \cdot 24$ & $7 \cdot 60$ \\
\hline$m$ & $1 \cdot 130$ & $1 \cdot 16$ & $1 \cdot 164$ & $1 \cdot 162$ & $1 \cdot 128$ & $1 \cdot 136$ & $1 \cdot 166$ & $1 \cdot 216$ \\
\hline
\end{tabular}

The values for $m$ are all greater than the theoretical value. There are also two values for each capillary according as the liquid flows in at one end or the other. This fact is most marked in the case of Capillary IV.

The above values for $\mathrm{C}$ were used in calculating $\mathrm{K}$ in equation (5) for the four tubes. $R / L$ was also calculated. These values are collected in the following table:-

\begin{tabular}{|c|c|c|c|c|}
\hline & Tube I. & Tube II. & Tube III. & Tube IV. \\
\hline K (meau) ...... & .005564 & .005463 & .005480 & .005478 \\
R/L ........... & .003407 & .002908 & .003774 & .003172 \\
\hline
\end{tabular}

It is evident that there is no linear relation between $K$ and $R / L$. When the above values are plotted in the way already mentioned, it will be noticed that they lie along a
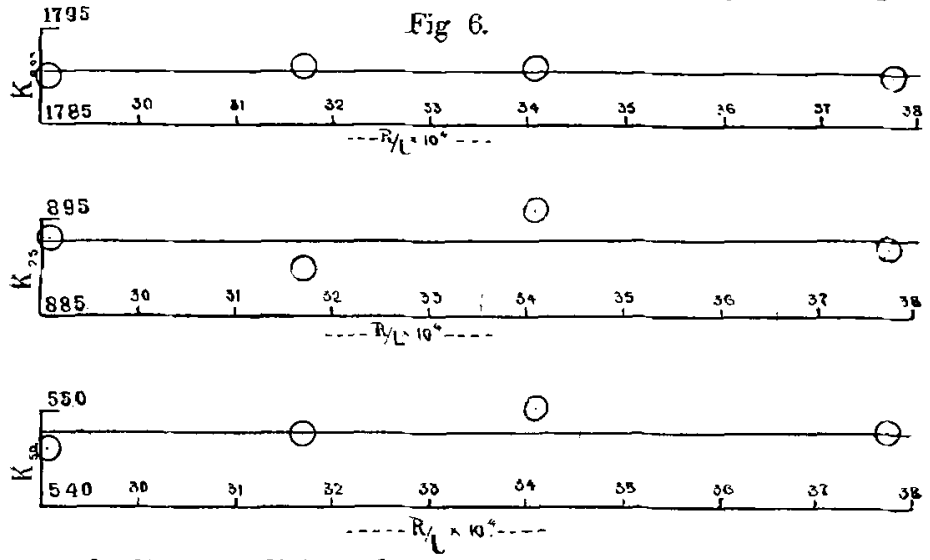

straight line parallel to the axis of abscissa ; that, therefore, $\mathrm{K}$ in equation (5) has zero value, i.e., the value for $n$ is zero. See fig. 6 
A set of readings was taken at $25^{\circ} \mathrm{C}$. and also at $0^{\circ} \mathrm{C}$., and values for $K_{2 \bar{s}}$ and $K_{0}$ were found. The results are tabulated below, and the corresponding graphs appear in fig. 6 . They bear out the conclusions arrived at in connexion with the results at $50^{\circ} \mathrm{C}$.

\begin{tabular}{|c|c|c|c|c|}
\hline & Tube I. & Tube II. & Tube III. & Tube IV. \\
\hline $\mathbf{K}(25 \cdot 0) \quad \ldots \ldots \ldots$ & $\cdot 00896$ & $\cdot 00893$ & .00892 & $\cdot 00890$ \\
\hline $\mathbf{K}_{\left(0^{\prime} 05\right)}$. & .01791 & .01790 & .01790 & 01791 \\
\hline $\mathrm{R} / \mathrm{L}$. & $\cdot 003407$ & $\cdot 002908$ & -003774 & $\cdot 003172$ \\
\hline
\end{tabular}

In the general reduction formula, the most difficult constant to measure accurately is $R$, the mean radius of efflux. Capillaries are not generally right circular cylinders, nor even elliptical cylinders; and as the degree of precision with which $R$ must be calculated is al ways four times as great as that required in the deduced viscosity, the examination and measurements of the capillaries must be carried on with extreme care.

Tubes I., II., III., and IV. were in the first place selected from a large number on account of their uniformity of bore-tested with a small mercury column-and their circular end sections.

The first method of measuring $\mathrm{R}$ was by contained volumes of mercury. The values obtained (at $0^{\circ}$ C.) for the mean radii were $\cdot 018968, \cdot 018926, \cdot 020416$, and $\cdot 020482 \mathrm{cms}$. respectively.

At the conclusion of all the experiments sections about $\frac{1}{2} \mathrm{~cm}$. in thickness were cut from the tubes at regular intervals ; they were ground, polished, and mounted in a brass plate. Three independent sets of readings of their dimensions were obtained by me-firstly, by direct comparison with a micrometer eyepiece in a microscope ; secondly, hy means of a microscope fitted to the dividing engine belonging to the Physics Laboratory, Melbourne University ; and, thirdly, by means of a micrometer microscope at the Sydney University. The following average values were obtained for the radii of the sections reduced to $0^{\circ} \mathrm{C}$ : :-

\begin{tabular}{|c|c|c|c|c|}
\hline $\begin{array}{l}\text { Capillary I. } \\
\text { (Cireular). }\end{array}$ & $\underset{\text { Method. }}{\text { First }}$ & $\begin{array}{l}\text { Second } \\
\text { Method. }\end{array}$ & $\begin{array}{c}\text { Third } \\
\text { Method. }\end{array}$ & $\begin{array}{l}\text { Mean } \\
\text { Values. }\end{array}$ \\
\hline Section 1 & $.01905 \mathrm{~cm}$ & $.01879 \mathrm{~cm}$ & $\cdot 01880$ & $\cdot 018838$ \\
\hline$" 2 \ldots$. & 01929 & $01919 \quad$ & .01899 & $\cdot 019106$ \\
\hline , 3 & ‘01932, & 01933 & .01877 & .019048 \\
\hline " 4 & 01926 & .01880, & .01881 & $\cdot 018882$ \\
\hline
\end{tabular}

[Mean radius (by mercury) $=\cdot 018968 \mathrm{~cm}$.] Mean $=018968 \mathrm{~cm}$. 


\begin{tabular}{|c|c|c|c|c|}
\hline $\begin{array}{l}\text { Capillary II. } \\
\text { (Circular). }\end{array}$ & $\begin{array}{c}\text { First } \\
\text { Method. }\end{array}$ & $\begin{array}{l}\text { Second } \\
\text { Method. }\end{array}$ & $\begin{array}{l}\text { Third } \\
\text { Method. }\end{array}$ & $\begin{array}{l}\text { Mean } \\
\text { Values. }\end{array}$ \\
\hline Section $1 \ldots .$. & $01899 \mathrm{~cm}$. & $\cdot 01889$ & $\cdot 01880$ & $\cdot 01880^{\circ} 2$ \\
\hline$" 2$. & 01896, & $\cdot 01861$ & 01880 & $\cdot 018763$ \\
\hline,$\quad 3 \ldots .$. & 01912 & $\cdot 01910$ & .01891 & .019009 \\
\hline \# 4 . & .01906, & .01909 & .01890 & $\cdot 018990$ \\
\hline
\end{tabular}

[Mean radius (by mercury) $=018926 \mathrm{~cm}$.] Mean $=018906 \mathrm{~cm}$.

\begin{tabular}{|c|c|c|c|c|}
\hline $\begin{array}{l}\text { Capillary III. } \\
\text { (Elliptical). }\end{array}$ & $\begin{array}{l}\text { First } \\
\text { Method. }\end{array}$ & $\begin{array}{l}\text { Second } \\
\text { Method. }\end{array}$ & $\begin{array}{c}\text { Third } \\
\text { Method. }\end{array}$ & $\begin{array}{l}\text { Mean } \\
\text { Values. }\end{array}$ \\
\hline & .02023 & '02066 & .02069 & $\cdot 020603$ \\
\hline \multirow[t]{2}{*}{ Section 1} & .02016 & $\cdot 01969$ & $\cdot 02020$ & .020023 \\
\hline & 02083 & $\cdot 02064$ & .02066 & $\cdot 020682$ \\
\hline & .02016 & .02009 & $\cdot 01993$ & 020022 \\
\hline & 02121 & 02119 & 02069 & $\cdot 020948$ \\
\hline \multirow[t]{2}{*}{$"$} & 02043 & .01983 & .01996 & $0 \cong 000$ \\
\hline & .02097 & 02032 & .02075 & 020643 \\
\hline$" \quad 4 \ldots .$. & .02043 & $\cdot 02010$ & .01998 & .020095 \\
\hline
\end{tabular}

Mean radius by mercury $=020416 . \quad \operatorname{Mear}_{1}\left\{\begin{array}{r}(a) \cdot 020719 \\ (b) \cdot 020035\end{array}\right.$

\begin{tabular}{|c|c|c|c|c|c|}
\hline \multicolumn{2}{|c|}{$\begin{array}{c}\text { Capillary IV. } \\
\text { (Elliptical). }\end{array}$} & $\begin{array}{c}\text { First } \\
\text { Method. }\end{array}$ & $\begin{array}{l}\text { Second } \\
\text { Method. }\end{array}$ & $\begin{array}{l}\text { Third } \\
\text { Method. }\end{array}$ & $\begin{array}{l}\text { Mean } \\
\text { Values. }\end{array}$ \\
\hline \multirow{3}{*}{ Section } & & 02138 & .02125 & .02088 & .021087 \\
\hline & 1 & .02023 & .02070 & .01976 & .020152 \\
\hline & & .02130 & .02118 & .02086 & 021040 \\
\hline \multirow[t]{2}{*}{ " } & $2 \ldots \ldots$ & .02043 & .02007 & .01984 & .020015 \\
\hline & & .02144 & $\cdot 02143$ & .02068 & .021057 \\
\hline & $3 \ldots \ldots$ & 02023 & $\cdot 01954$ & $\cdot 01976$ & $\cdot 019765$ \\
\hline & & .02183 & .02125 & 02079 & 021042 \\
\hline & $4 \ldots \ldots$ & -02023 & .01997 & .01994 & 019723 \\
\hline & & & & ing & $\begin{array}{l}021056 \\
(b) \cdot 019914\end{array}$ \\
\hline
\end{tabular}

In determining the mean value for each section, the values obtained by the three methods were weighted in the following manner : Method 1, weight 1; Method 2, weight 2; Method 3 , weight 3 ; thus for Capillary 1 , Section 1 , by adding. 
logether $\cdot 01905 \mathrm{~cm}$., twice $\cdot 01879 \mathrm{~cm}$, and thres times $-01880 \mathrm{~cm}$., and dividing the sum by 6 , we obtain the value $.018838 \mathrm{~cm}$. The mean obtained in this way is, I consider, the best value the individual results will produce, taking in account the experimental difficulties in ineasuring such small bores in the three cases.

The mean values by neasurement were then combined with the values obtained by mercury column, and in this way the final values were obtained.

Capillary I., circular cylinder, radius $018968 \mathrm{~cm}$. at $0^{\circ} \mathrm{C}$.

$$
\begin{aligned}
& \text {," III., elliptical cylinder, semi-axes }(a) \cdot 020762, \\
& \text { (b) } \cdot 020076 \text { ", } \\
& . \quad \text { IV., , , }, \quad, \quad \text { (a) } 021061, \\
& \text { (b) } 019919, \\
& \text {, III., mean radius of efflux } \mathrm{R}\left(\text { where } \mathrm{R}^{4}=\frac{2 a^{3} b^{3}}{a^{2}+b^{2}}\right) \\
& =\cdot 02041 \dot{\mathrm{cm}} \text {. } \\
& \text {, IV., mean radius of efflux } \mathrm{R}=-020474 \mathrm{~cm} \text {. }
\end{aligned}
$$

An accuracy of 1 in 1000 was aimed at throughout the experiments. The values for the constants in the reduction formula having been obtained, the viscosity was determined from the various observations which had been made from time to time, including those already mentioned.

A special set of experiments at $0^{\circ} \mathrm{C}$. was made at a fixed pressure in order to obtain accurate values of the viscosity at $0^{\circ} \mathrm{C}$. The thermometer registered $0^{\circ} 05 \mathrm{C}$. throughout the series. With Tube I. in the glischrometer, under a pressure of $197 \mathrm{cms}$. of water, the average time of flow was 109.7 secs. The first term of the reduction formula was found to be $\cdot 018690$, and the second term 000769 . The value for the viscosity at $0^{\circ} 05$ was 01792 ; which reduced to $0^{\circ} \mathrm{C}$, becomes 01795 . A second determination gave the same values practically. The observations and reductions are given on p. 513 , also a similar set at $25^{\circ} \mathrm{C}$. p. 514 .

Most of the experiments, however, were carried out at $50^{\circ} \mathrm{C}$. with the water flowing under different pressures. In the following tables (pp. 515-517) are collected the various results. The pressures are given in centimetres of mercury, the times of flow in seconds, the values of viscasity (double observations) in absolute measure, and the kinetic energy correction-second term-also in absolute measure. The pressures and times are approximate, and the viscosity values are reduced to the even temperature $50^{\circ} \mathrm{C}$. 
Viscosity of Water.

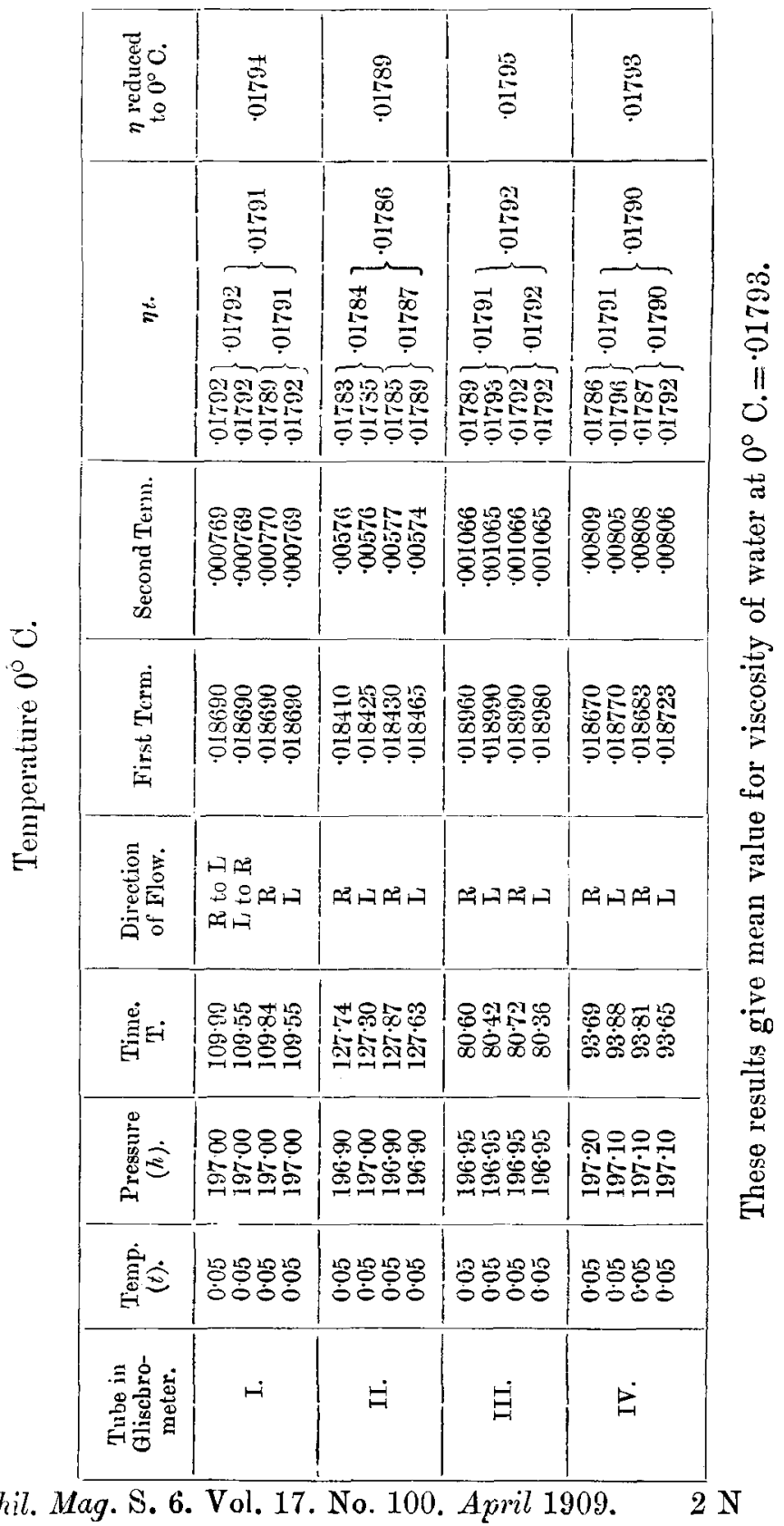




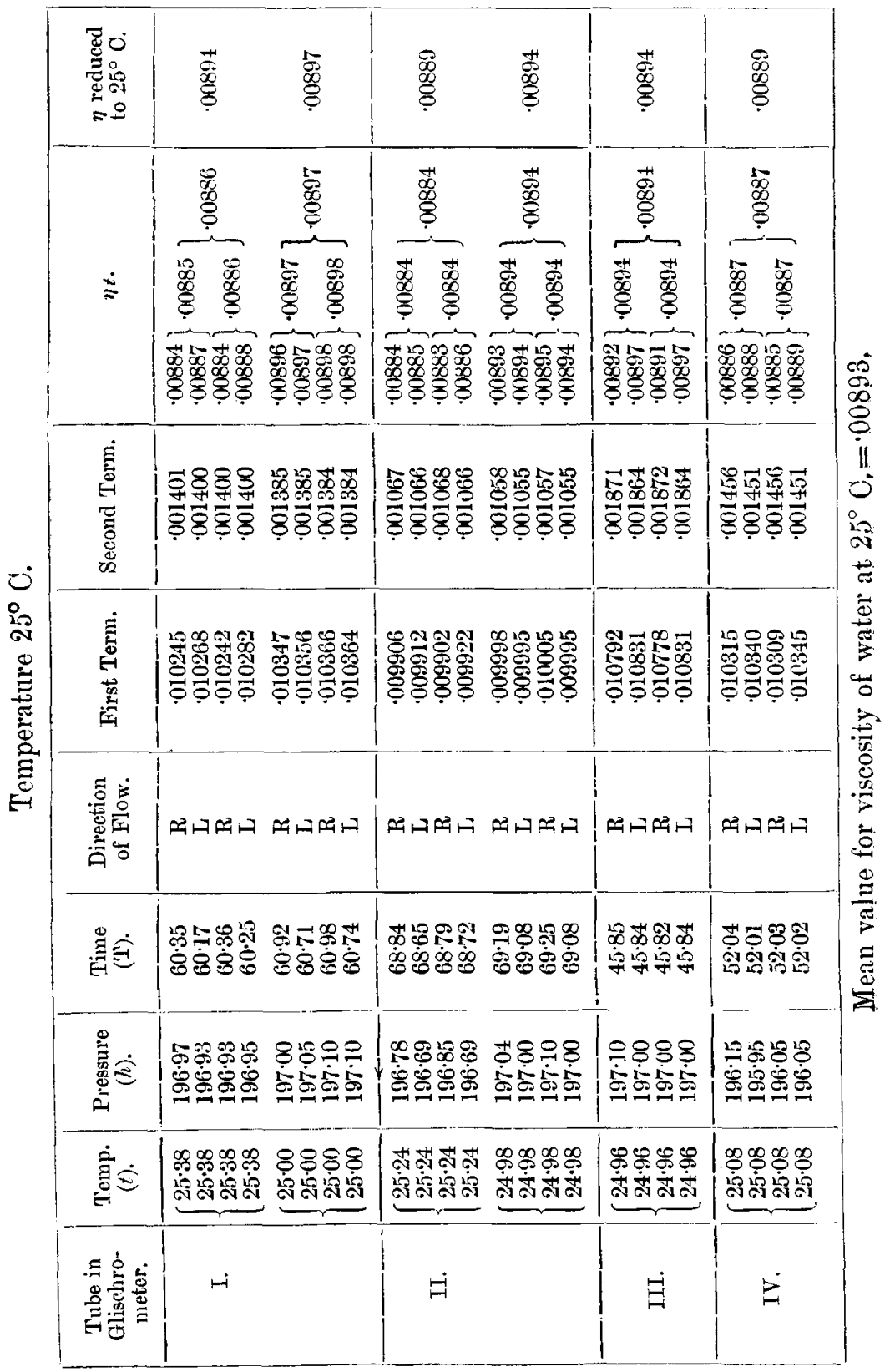


Viscosity of Water.

Results at $50^{\circ} \mathrm{C}$. with Tube I. in Glischrometer.

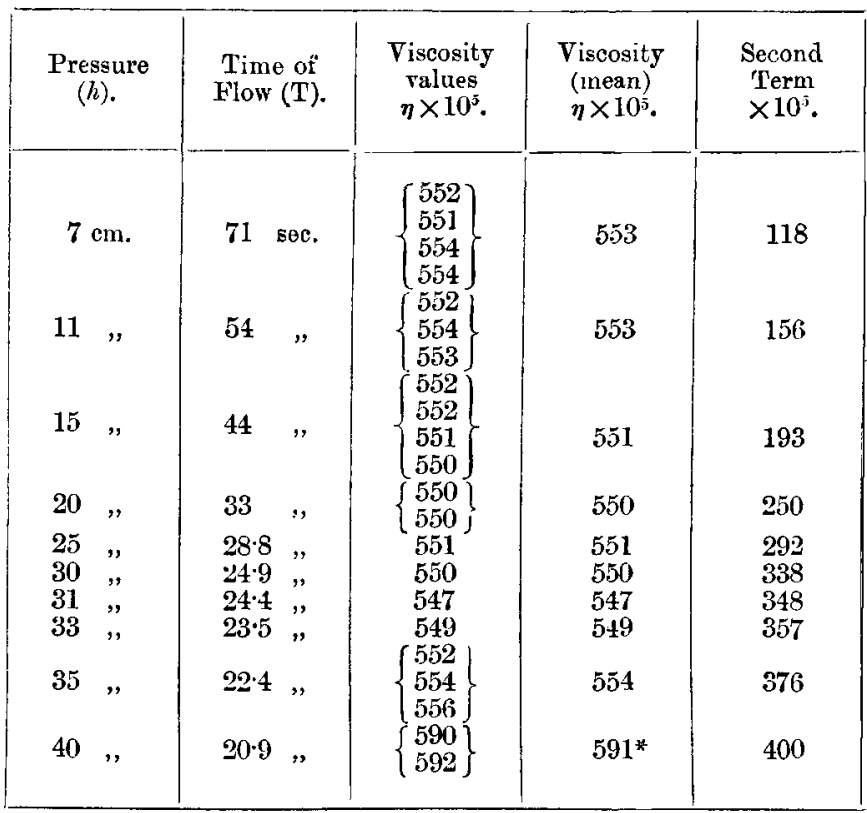

Results at $50^{\circ}$ C. with Tube II. in Glischrometer.

\begin{tabular}{|c|c|c|c|c|c|c|}
\hline \multicolumn{2}{|c|}{$\begin{array}{c}\text { Pressure } \\
(h) \\
\text { (Approx.). }\end{array}$} & \multicolumn{2}{|c|}{$\begin{array}{c}\text { Time of } \\
\text { Flow (T) } \\
\text { (Approx.). }\end{array}$} & $\begin{array}{c}\text { Second } \\
\text { Term } \\
\times 10^{-} \text {. }\end{array}$ & $\begin{array}{c}\text { Viscosity } \\
\times 10^{5}\end{array}$ & $\begin{array}{c}\text { Mean } \\
\nabla \text { iscosity } \\
\times 10^{5} .\end{array}$ \\
\hline \multicolumn{2}{|c|}{$7.3 \mathrm{~cm}$} & \multicolumn{2}{|c|}{80.5 see. } & 91 & $\left\{\begin{array}{l}549 \\
550\end{array}\right\}$ & 550 \\
\hline 11 & , & 603 & $"$ & 121 & $\left\{\begin{array}{l}549 \\
549 \\
549\end{array}\right.$ & 549 \\
\hline 15 & $"$ & $48 \cdot 3$ & ", & 151 & $\left\{\begin{array}{l}549 \\
548\end{array}\right\}$ & 548 \\
\hline 20 & $"$ & $36 \cdot 8$ & , & 199 & $\left\{\begin{array}{l}549 \\
548\end{array}\right\}$ & 549 \\
\hline 30 & $"$ & 27 & , & 269 & $\left\{\begin{array}{l}553 \\
553\end{array}\right\}$ & 553 \\
\hline 40 & , & 23 & $"$ & 316 & $\left\{\begin{array}{l}557 \\
561\end{array}\right\}$ & $559 *$ \\
\hline 42 & , & $21 \cdot 5$ & , & 340 & 565 & $565 *$ \\
\hline
\end{tabular}

Mean $\cdot 00550$

$2 \mathrm{~N} 2$ 
With Tube I. in the glischrometer, the formula gives constant valnes up to a pressure of $35.4 \mathrm{cms}$. of mercury, when the time of flow is about 22 seconds. It will be noticed that at this point the kinetic energy correction is more than 60 per cent. of the viscosity, and the average velocity in the tube is $400 \mathrm{cms}$. per sec.

In the case of Tube II. the formula breaks down suddenly at a pressure between $35.7 \mathrm{cms}$. and $36.9 \mathrm{cms}$, the time of flow being about 24 secs., and the correction at pressure 35.7 cms. more than 55 per cent. of the viscosity. The velocity in this case is 370 cms. per sec.

Results at $50^{\circ}$ C. with Tube III. in Glischrometer.

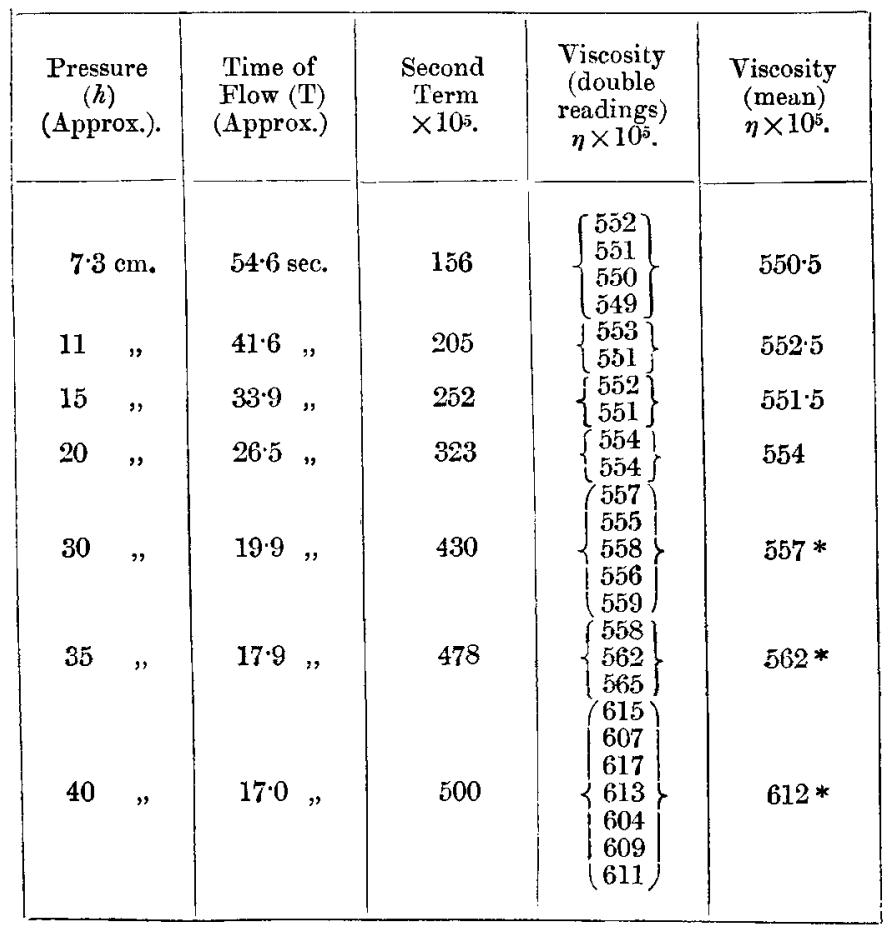

Mean $\cdot 00552$

For Tube III. the figures are, pressure between $20.4 \mathrm{cms}$. and $30.4 \mathrm{cms}$., time of flow less than 26 secs., second term 
60 per cent. of the viscosity; and for Tube IV. when the pressure is $27 \mathrm{cms}$. and the second term is nearly 60 per cent. of the viscosity, the deduced value is satisfactory, but an increase of pressure of $1 \mathrm{~cm}$. brings about some decided change. The highest velocity reached in both cases, before

Results at $50^{\circ} \mathrm{C}$. with Tube IV. in Glischrometer.

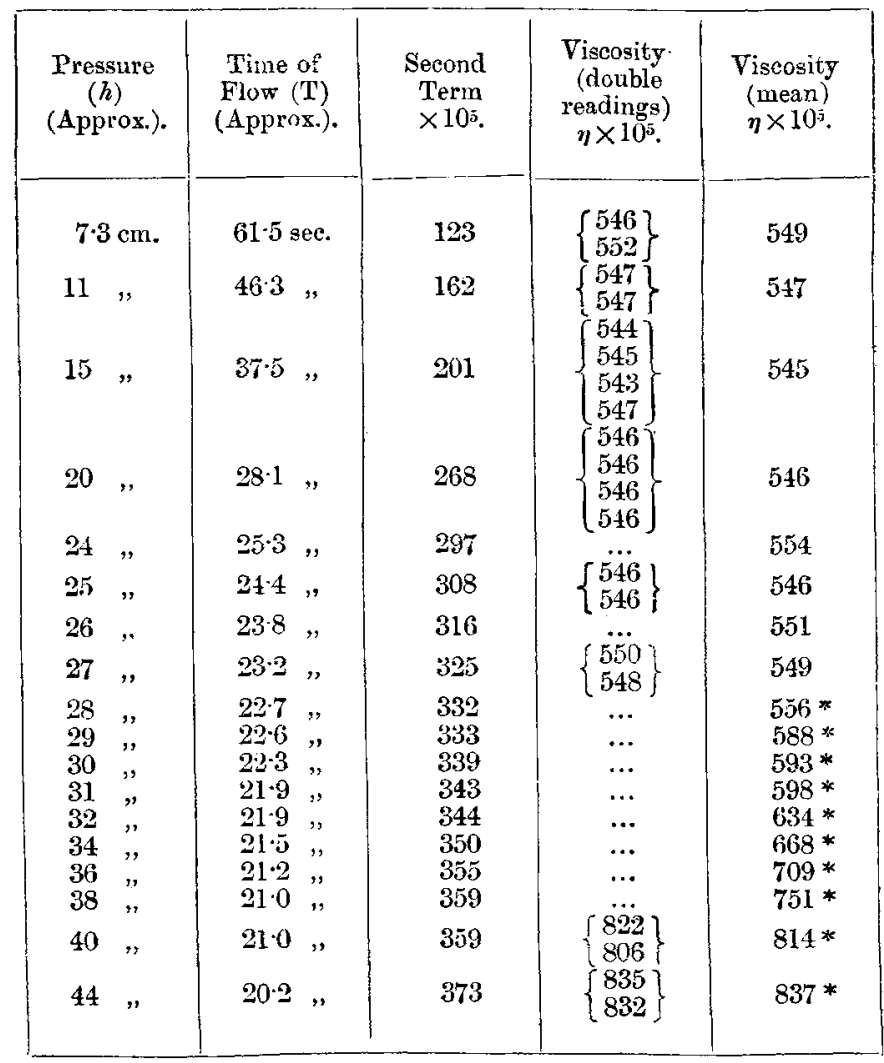

Mean 00548

the change, was about 340 cms. per sec. The curves in fig. 5 (Pl. XIII.) indicate that when the change takes place, there is a large increase in the value of $m$, if the formula still holds; but the individual results do not agree sufficiently well to enable one to draw definite conclusions from them. 
Viscosity Values $\left(\times 10^{5}\right)$ at $50^{\circ}$ C. collected.

\begin{tabular}{|c|c|c|c|c|c|c|}
\hline \multicolumn{2}{|c|}{ Pressure. } & Tube I. & Tube II. & Tube III. & Tube IV. & A verage. \\
\hline \multicolumn{2}{|c|}{$7 \cdot 3 \mathrm{~cm}$} & 553 & 550 & 551 & 549 & 551 \\
\hline \multicolumn{2}{|c|}{$11 \cdot 0 "$} & 553 & 549 & 552 & 547 & 550 \\
\hline \multirow{2}{*}{\multicolumn{2}{|c|}{$\begin{array}{l}14 \cdot 7 " \\
20 \%\end{array}$}} & 551 & 548 & 552 & 545 & 549 \\
\hline & & 550 & 549 & 554 & 546 & 550 \\
\hline \multirow{2}{*}{\multicolumn{2}{|c|}{$\begin{array}{l}24 \\
25\end{array}$}} & & $\ldots$ & $\ldots$ & 554 & 554 \\
\hline & & 551 & ... & $\cdots$ & 546 & 549 \\
\hline \multicolumn{2}{|c|}{$26 \quad "$} & & $\ldots$ & $\ldots$ & 551 & 551 \\
\hline \multirow{2}{*}{\multicolumn{2}{|c|}{$\begin{array}{l}27 \\
29 \quad "\end{array}$}} & 550 & $\ldots$ & $\ldots$ & 549 & 550 \\
\hline & & 551 & $\because$ & & $(588)$ & 551 \\
\hline \multirow{2}{*}{\multicolumn{2}{|c|}{$\begin{array}{l}30 \quad ", \\
31\end{array}$}} & 550 & 553 & (557) & (593) & 551 \\
\hline & & 547 & $\ldots$ & $\ldots$ & $(598)$ & 547 \\
\hline \multicolumn{2}{|c|}{$33 "$} & 549 & $\ldots$ & $\ldots$ & & 549 \\
\hline \multirow{2}{*}{\multicolumn{2}{|c|}{$35 \quad "$}} & 551 & $\ldots$ & . & $(668)$ & 551 \\
\hline & & 554 & $\ldots$ & $(562)$ & & 554 \\
\hline & " & & $\cdots$ & $(574)$ & (709) & \\
\hline & ", & $(558)$ & $\cdots$ & $(571)$ & $(754)$ & \\
\hline & $"$ & $(573)$ & $\cdots$ & $\begin{array}{l}(578) \\
(599)\end{array}$ & $(751)$ & \\
\hline & $"$ & (591) & (559) & (612) & (8i4) & \\
\hline $\begin{array}{l}40 \\
42 \\
43\end{array}$ & $"$ & $\cdots$ & $(565)$ & $\cdots$ & & \\
\hline 43 & $"$ & $\ldots$ & $\cdots$ & $\cdots$ & $(\varepsilon 37)$ & \\
\hline \multicolumn{2}{|c|}{ Average $=$} & $\cdot 00551$ & $\cdot 00550$ & $\cdot 00552$ & $\cdot 00548$ & \\
\hline
\end{tabular}

Mean Value $=00550$

The values for the viscosity of water at $50^{\circ} \mathrm{C}$, obtained with the various capillary tubes in the glischrometer, are collected in the foregoing table. The mean value is $\cdot 00550$.

\section{Results.}

(1) The constants in the reduction formula were all determined with the greatest possible degree of accuracy, including $\mathrm{R}, n$, and $m$.

(2) For each capillary in the glischrometer-four were used separately - two values for $m$ were found, one for each of the ends. These values were in every case greater than the theoretical value $1 \cdot 12$.

(3) For the series of capillary tubes used, experiments at temperatures $0^{\circ}$ C., $25^{\circ}$ C., and $50^{\circ} \mathrm{C}$. gave in each case zero values for $n$.

(4) Absolute values for the viscosity of water at $0^{\circ} \mathrm{C}$, $26^{\circ} \mathrm{C}$, and $50^{\circ} \mathrm{C}$. were obtained, namely, $\cdot 01793, \cdot 00893$, and $\cdot 00550$; which are probably correct to $0 \cdot 1$ per cent. 
(5) The values obtained for $m$ were constant over a big range of pressure; and at a very high pressure there was an indication of an abrupt change in the value of $m$, or in the nature of the flow. The velocities at this pressure were much below the critical velocities for the various tubes, but were all above the lower limit of critical velocity.

(6) Consistent values for the viscosity of water at $50^{\circ} \mathrm{C}$. were obtained in cases where the kinetic energy correction was as high as 60 per cent. of the viscosity.

I have much pleasure in acknowledging my indebtedness to Professor Sir J. J. Thomson, Cavendish Laboratory, Cambridge ; Professor Luyle, Melbourne University; Professor Pollock, Sydney University ; and Mr. G. H. Knibbs, F.R.A.S., Federal Statistician, formerly Director of Technical Education, N.S.W., and Lecturer in Surveying, University of Sydney, for valuable assistance during the progress of this research, which was commenced at the Cavendish Laboratory, Cambridge, and completed at the Sydney University.

Note on the Viscosity of Solutions. - The viscosity of certain lithium chloride solutions was determined with the glischrometer described in the previous paper. The only novel feature of the measurements was the automatic recording of the time of flow. The inner platinum wires at $b$ and $c$ (fig. 1, p. 503) were connected by insulated wires, also the inner wires at $a$ and $d$. Wires were fastened to the outer wires at $a, b, c$, and $d$, and were connected to four plugs on a double reversing key. The two remaining plugs were joined by wires through a battery and one of the electromagnets already described. With the key in one position, there was electrical communication between the battery and electromagnet and the outer $c$ on the one side, and the outer $b$ on the other side. The circuit was complete only when the solution filled the spaces at both $c$ and $b$. With the key reversed, the battery was connected to the outer $a$ and the outer $a$, and the circuit was complete when the solution filled the spaces at $a$ and $d$. By regulating the amount of solution in the glischrometer the signals could be made as short as necessary, at the beginning and end of the flow from $\mathrm{R}$ to $\mathrm{L}$, or in the opposite direction, and the time of flow could be read off accurately on the tape.

The following set of readings will be sufficient to illustrate 
the accuracy with which determinations of the viscosity of solutions can be made with this arrangement:-

Lithium Chloride Solution, Temperature $20^{\circ} .75 \mathrm{C}$.

\begin{tabular}{|c|c|c|c|c|}
\hline $\begin{array}{c}\text { Pressure } \\
(h)\end{array}$ & $\begin{array}{c}\underset{(\mathrm{T})}{\operatorname{Time}} \mathrm{.} \\
\end{array}$ & $\begin{array}{c}\text { Correction. } \\
\times 10^{3}\end{array}$ & $\eta$ & $\begin{array}{c}\eta \\
\text { (mean) }\end{array}$ \\
\hline $\begin{array}{l}\left\{\begin{array}{l}198 \cdot 0 \mathrm{~cm} . \\
1982 \\
179 \cdot 3 \\
179 \cdot 1 \\
151 \cdot 0 \\
151 \cdot 2\end{array},\right. \\
\left\{\begin{array}{l}127 \cdot 1 \\
127 \cdot 0 \\
100 \cdot 5 \\
100 \cdot 0 "\end{array}\right. \\
\text {, ", }\end{array}$ & $\begin{aligned} 57 \cdot 2 \text { sec. } \\
56 \cdot 4 \quad " \\
62 \cdot 8 \quad " \\
62 \cdot 8 \quad " \\
73 \cdot 1 \quad " \\
73 \cdot 0 " \\
85 \cdot 6 \quad " \\
85 \cdot 1 \quad " \\
106 \cdot 6 \quad " \\
106 \cdot 8 \quad "\end{aligned}$ & $\begin{array}{r}150 \\
151 \\
136 \\
138 \\
118 \\
118 \\
101 \\
101 \\
81 \\
81\end{array}$ & $\left.\begin{array}{l}\cdot 01201 \\
\cdot 01198 \\
\cdot 01204 \\
\cdot 01197 \\
\cdot 01200 \\
\cdot 01200 \\
\cdot 01197 \\
\cdot 01198 \\
\cdot 01200 \\
\cdot 01203\end{array}\right\}$ & $\begin{array}{l}.01200 \\
.01200 \\
.01200 \\
.01198 \\
.01201\end{array}$ \\
\hline
\end{tabular}

A verage $\cdot 01200$

XLVII. The Doppler Effect in Positive Rays. By John Trowbridge *.

\section{[Plate XIV.]}

THE discovery of canal rays by Goldstein, and that of the Doppler effect in these rays, marks an epoch in the study of the discharge of electricity through gases; for before these discoveries the multitude of confusing effects which arise in the space between the anode and the cathode made it difficult to observe any translation movements. The space, however, behind the cathode is comparatively free for the passage of the positive ions.

We now recognize, in addition to the positive rays befind the cathode-the canal rays-retrograde positive rays which are directed to the anode, or rather away from the cathode in the direction of the anode $\dagger$.

This later discovery leads one to expect that the Doppler effect should be found also between the anode and the cathode. The result of $\mathrm{my}$ study shows that the effect does exist in this region and indicates a movement away from the cathode and toward the anode.

* Communicated by the Author.

† Wehnelt, Wied. Am. A. 1899, p. 421; Runge \& Paschen, Wied. Ann. lxi. 1897, p. 644; Paschen, Wied. Ann. xxiii. 1907, p. 247 Villard, Comptes Rendus, cxliii. 1906, p. 673; Goldstein, Phil. Mag. March 1908, p. 372; Jacob Kunz, Phil. Mag. July 1908, p. 161; J. J Thomson, Phil. Mag. Oct. 1908, p. $6 \overline{7}$. 
Fig. 2.

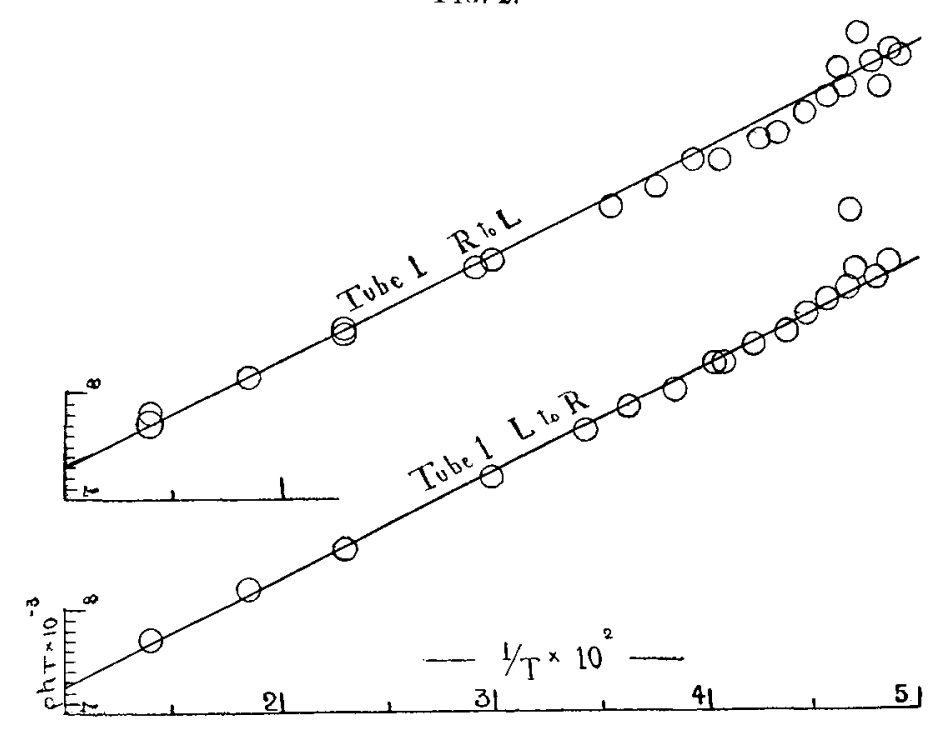

FIG. 4.

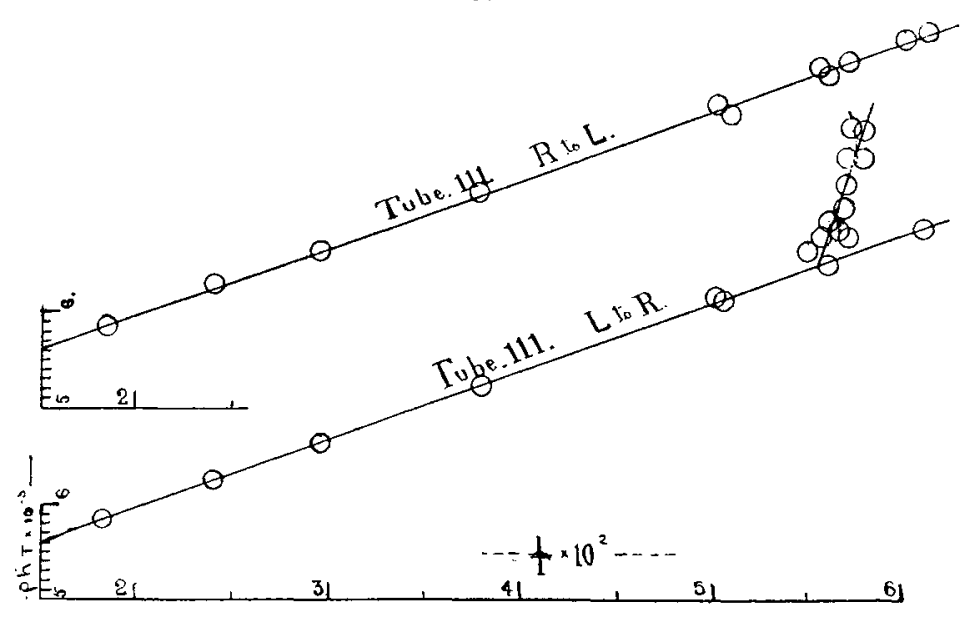

FI*. 3.

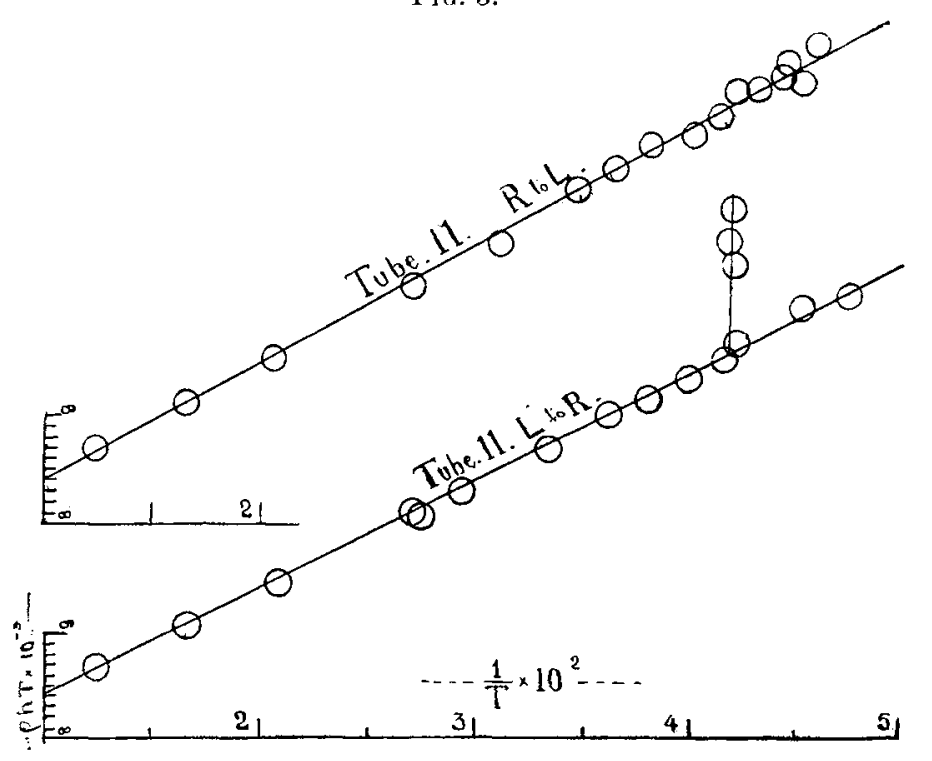

Frg. 5.

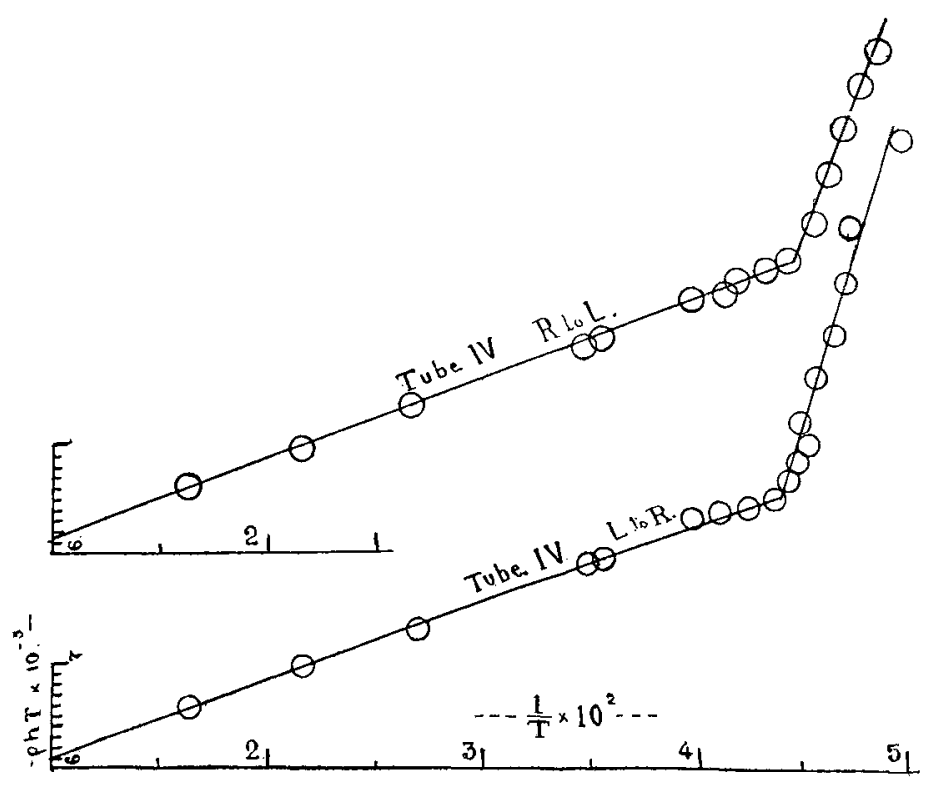

